\title{
A nomogram to predict survival in non-small (1) cell lung cancer patients treated with nivolumab
}

\author{
Andrea Botticelli ${ }^{1}$, Massimiliano Salati ${ }^{2,3^{*}}$, Francesca Romana Di Pietro ${ }^{1}$, Lidia Strigari ${ }^{4}$, Bruna Cerbelli ${ }^{5}$, \\ Ilaria Grazia Zizzari ${ }^{6}$, Raffaele Giusti ${ }^{1}$, Marco Mazzotta ${ }^{1}$, Federica Mazzuca' ${ }^{1}$ Michela Roberto ${ }^{1}$, Patrizia Vici ${ }^{7}$, \\ Laura Pizzuti ${ }^{7}$, Marianna Nuti ${ }^{6}$ and Paolo Marchetti ${ }^{1}$
}

\begin{abstract}
Background: The advent of immune checkpoint inhibitors ( $\mathrm{ICls}$ ) has considerably expanded the armamentarium against non-small cell lung cancer (NSCLC) contributing to reshaping treatment paradigms in the advanced disease setting. While promising tissue- and plasma-based biomarkers are under investigation, no reliable predictive factor is currently available to aid in treatment selection.

Methods: Patients with stage IIIB-IV NSCLC receiving nivolumab at Sant'Andrea Hospital and Regina Elena National Cancer Institute from June 2016 to July 2017 were enrolled onto this study. Major clinicopathological parameters were retrieved and correlated with patients' survival outcomes in order to assess their prognostic value and build a useful tool to assist in the decision making process.

Results: A total of 102 patients were included in this study. The median age was 69 years (range 44-85 years), 69 (68\%) were male and 52\% had ECOG PS 0. Loco-regional/distant lymph nodes were the most commonly involved site of metastasis (71\%), followed by lung parenchyma (67\%) and bone (26\%). Overall survival (OS) in the whole patients' population was $83.6 \%, 63.2 \%$ and $46.9 \%$ at 3,6 and 12 months, respectively; while progression-free survival (PFS) was $66.5 \%, 44.4 \%$ and $26.4 \%$ at 3,6 and 12 months, respectively. At univariate analysis, age $\geq 69$ years $(P=0.057)$, ECOG PS $(P<0.001)$, the presence of liver $(P<0.001)$, lung $(P=0.017)$ metastases, lymph nodes only involvement $(P=0.0145)$ were significantly associated with OS and ECOG PS $(P<0.001)$ and liver metastases $(P<0.001)$, retained statistical significance at multivariate analysis. A prognostic nomogram based on three variables (liver and lung metastases and ECOG PS) was built to assign survival probability at 3, 6, and 12 months after nivolumab treatment commencement.

Conclusion: We developed a nomogram based on easily available and inexpensive clinical factors showing a good performance in predicting individual OS probability among NSCLC patients treated with nivolumab. This prognostic device could be valuable to clinicians in more accurately driving treatment decision in daily practice as well as enrollment onto clinical trials.
\end{abstract}

Keywords: Immunotherapy, Lung cancer, Prognostic factors, Nivolumab, Nomogram

\footnotetext{
*Correspondence: maxsalati@live.it

${ }^{2}$ Department of Oncology, University Hospital of Modena and Reggio

Emilia, Modena, Italy

Full list of author information is available at the end of the article
} 


\section{Background}

Lung cancer represents a massive health burden worldwide with 1.7 million deaths annually and a $26 \%$ increase in incidence during the last decade [1]. More than a half of patients present with stage IV disease and less than $5 \%$ of them survive beyond 5 years [2].

The introduction of immune checkpoint inhibitors (ICI) has considerably expanded the armamentarium against non-small cell lung cancer (NSCLC) contributing to reshaping treatment paradigms in the advanced disease setting $[3,4]$. The anti-PD-1 pembrolizumab both as monotherapy and combined with platinum/pemetrexed doublet is considered a first-line treatment option in PD-L1 overexpressing ( $\geq 50 \%)$ [5] and unselected patients [6], respectively, in absence of actionable oncogenic drivers. Moreover, the anti-PD-L1 atezolizumab has emerged as a further front-line therapeutic choice both in combination with bevacizumab, carboplatin and paclitaxel [7] and platinum-based doublets [8] in NSCLC regardless of PD-L1 status. In the second-line setting, nivolumab [9] and atezolizumab [10] (irrespective of PD-L1 expression) and pembrolizumab (PD-L1 $\geq 1 \%$ ) [11] are approved as single-agent for chemotherapy pretreated, immunotherapy-naïve patients.

The fast-growing number of immunotherapeutics and their limited efficacy with $70-80 \%$ of patients progressing within the first 2-3 months underline the need for predictive biomarkers aiding in treatment selection [12]. Moreover, a subset of patients termed as hyperprogressors and ranging from 9 to $29 \%$ have been described that experience a paradoxically accelerated tumour growth while on ICI treatment [13]. Tumour-associated macrophages reprogramming towards a pro-tumorigenic phenotype upon Fc receptor engagement by ICI has been suggested to have a causative role in this phenomenon in patients with distinctive immune and genetic profiles.

Nivolumab is a fully human IgG4 anti-PD1 monoclonal antibody that showed to prolong OS compared to docetaxel in NSCLC failing first-line chemotherapy. However, it yielded a response rate as low as $13.6 \%$ to $23 \%$ and a median PFS of 2.3 to 4 months in biomarker-unselected patients $[9,10]$. Several biomarkers are being studied that can help to enrich for patients more likely to benefit from nivolumab $[14,15]$. PD-L1 is a suboptimal predictive biomarker since less than 50\% of PD-L1-selected patients respond to treatment and some responders may be encountered also in 'biomarker-negative' cohorts. Tumour mutational burden (TMB) holds great promise and up to now is the sole clinically validated biomarker. Nevertheless, no consensus exists on how it should be measured and its widespread use is thus limited. Additional promising tissue- and plasma-based predictive biomarkers are under investigation, including tumour infiltrating lymphocytes, "immunoscore" (composite biomarker integrating four $\mathrm{T}$ cell related IHC features), immune gene signatures, eosinophil, lymphocyte and neutrophil counts and relative ratios from peripheral blood, plasma IL-6 and IDO, microsatellite instability status, interferon signature, $\mathrm{T}$ cell repertoire, MHC status and microbiome profile [14]. Among clinical factors, poor performance status (ECOG PS $\geq 2$ ), a period of time since prior treatment $\geq 6$ months and involvement of more than one metastatic site have been independently associated with shorter OS in a cohort of 175 pretreated NSCLC patients receiving nivolumab [16]. More recently, ECOG PS $\geq 2$, liver and lung metastases have been suggested to be independent predictors of nivolumab efficacy in an Asian population of 201 advanced NSCLC [17].

The aim of our study is to assess the predictive-prognostic significance of clinicopathological parameters in NSCLC patients receiving second-line nivolumab treatment in clinical practice in order to build a useful tool to assist in the decision making process.

\section{Materials and methods \\ Patients}

Patients with stage IIIB-IV NSCLC receiving nivolumab at Sant'Andrea Hospital and Regina Elena National Cancer Institute from June 2016 to July 2017 were enrolled onto this study. Inclusion criteria were: age $>18$ years; histologically-documented diagnosis of NSCLC; Eastern Cooperative Oncology Group (ECOG) performance status $\leq 2$; measurable disease; progression on or after first-line platinum-containing doublet; patients harboring oncogenic driver aberrations (i.e. EGFR mutations or ALK fusion oncogene) were required to have received previous tyrosine kinase inhibitor therapy; adequate cardiac, pulmonary, renal, liver and bone marrow function; patients with stable and asymptomatic central nervous system metastases were eligible. Exclusion criteria were: autoimmune disease; symptomatic interstitial lung disease and any other significant comorbidity; systemic immunosuppression; prior treatment with immune-stimulatory antitumor agents including checkpoint-targeted agents. All patients gave written informed consent.

The study was conducted in accordance with good clinical practice guidelines and the declaration of Helsinki. The final version of the protocol was approved by the Institutional Ethics Committee of the two Institutions involved.

\section{Treatment, efficacy and safety assessments}

Nivolumab was administered intravenously at a standard dose of $3 \mathrm{mg} / \mathrm{kg}$ every 2 weeks until disease progression or development of unacceptable toxicity. Tumour 
response was assessed at week 9 and every 6 weeks thereafter until disease progression using immune-related Response Evaluation Criteria in Solid Tumors Criteria (i-RECIST) and classified according to disease control (complete response, partial response and stable disease) and progressive disease. Safety assessments were performed at day 1 of every cycle until the end of treatment and toxicities were graded according to the National Cancer Institute Common Terminology Criteria for Adverse Events (version 4.0).

\section{Objectives and outcomes}

Progression-free survival (PFS) was defined as the time from nivolumab commencement until the first documented tumour progression or death from any cause, whichever occurred first. Overall survival (OS) was defined as the time from nivolumab commencement to death from any cause.

Early progressors patients were defined as those experiencing disease progression within 3 months from the beginning of nivolumab treatment.

The association between early progression and clinicopathological factor PS, age, sex and site of metastases was assessed together with the association between PFS > 12 months and PS, age, sex and site of metastases.

\section{Statistical analysis}

Categorical variables are presented as a number with a percentage in descriptive tables, and they were compared with Fisher's exact test or Pearson's Chi-square test. The impact of clinicopathological variables on overall survival (OS) and progression-free survival (PFS) was analyzed by both the univariate and multivariate analyses (UVA and MVA, respectively). With regards to UVA, patients' OS and PFS were analyzed using the Kaplan-Meier method and log-rank tests. Prognostic clinic-pathological variables deemed of potential relevance in the univariate analysis (corresponding to a cutoff of $\mathrm{P}<0.10$ ) were included in the multivariate Cox proportional hazards regression analysis. A nomogram to predict 3, 6, and 12-months survival probability was developed based on covariates retaining a statistically significant power $(P<0.05)$ in MVA. To quantify the discrimination performance of the nomogram, Harrell's C-index was measured. The nomogram was subjected to bootstrapping validation (1000 bootstrap resamples) to calculate a relatively corrected C-index. Calibration was studied graphically after grouping patients into deciles with respect to their predicted probabilities and plotting the mean predicted probabilities against the mean observed probabilities. Bootstrapping was applied to correct the model based on the estimated optimism. Internal validation was performed determining the OS in each calculated group. Discrimination of nomogram was tested by Kaplan-Meier curves and boxplots. A $P<0.05$ was considered statistically significant. Statistical analyses were performed using R-package software.

\section{Results \\ Patients}

A total of 102 patients fulfilled the inclusion criteria and were enrolled in this study. Overall, the median age was 69 years (range $44-85$ years), Sixty-nine (68\%) were male and $52 \%$ had ECOG PS 0. Loco-regional/distant lymph nodes were the most commonly involved site of metastasis (71\%), followed by lung parenchyma (67\%) and bone (26\%). Other baseline clinicopathological parameters are reported in Table 1. Seventy-two (88\%) patients experienced early progression, while seven (39\%) patients presented PFS longer than 12 months.

After a median follow-up period of 11 months (range 1-29 months), all 102 treated patients were assessable for OS. At the time of the analysis, 55 (54\%) patients had died. OS in the whole patients' population was $83.6 \%, 63.2 \%$ and $46.9 \%$ at 3,6 and 12 months, respectively; while PFS was $66.5 \%, 44.4 \%$ and $26.4 \%$ at 3,6 and 12 months, respectively.

\section{Prognostic factors, nomogram development and performance}

The OS was significantly shorter in patients aged $>69$ years old compared to those younger than 69 years old. Patients with liver metastases (Figs. 1, 2) experienced a significantly lower OS, while those affected by lung metastatic deposits lived longer.

At UVA assuming the cutoff of $\mathrm{P}<0.10$ for include potential interest parameters, age $\geq 69$ years $(P=0.057)$, ECOG PS $(P<0.001)$, the presence of liver $(P<0.001)$, lung $(P=0.017)$ metastases, lymph nodes only involvement $(P=0.0145)$ were significantly associated with OS and were included in the multivariate analysis. ECOG PS $(P<0.001)$ and liver metastases $(P<0.001)$, retained statistical significance at MVA (Tables 2, 3). Based on the estimated regression coefficients in the Cox analysis, a prognostic nomogram that included liver, lung metastases and ECOG PS was developed to assign survival probability at 3, 6, and 12 months after nivolumab treatment commencement (Fig. 3).

To use the nomogram, a vertical line needs to be delineated to the point raw to assign point values for each variable. Thereafter, the corresponding points are to be summed to obtain the total points. Finally, from the total points a vertical line needs to be drawn to get the value of 3, 6, 12 months OS probability. The presence of lung 
Table 1 Baseline clinico-pathological characteristics $(n=102)$

\begin{tabular}{|c|c|}
\hline Parameter & N (\%) \\
\hline Age years (median, range) & $69(44-85)$ \\
\hline Height & $168 \mathrm{~cm}(152-186$ \\
\hline Weight & $69.5 \mathrm{~kg}(45-175)$ \\
\hline \multicolumn{2}{|l|}{ Gender } \\
\hline Male & $69(68 \%)$ \\
\hline Female & $33(32 \%)$ \\
\hline \multicolumn{2}{|l|}{ ECOG PS } \\
\hline 0 & $53(52 \%)$ \\
\hline 1 & $41(40 \%)$ \\
\hline 2 & $8(8 \%)$ \\
\hline \multicolumn{2}{|l|}{ Lung parenchyma metastasis } \\
\hline Yes & $68(67 \%)$ \\
\hline No & $34(34 \%)$ \\
\hline \multicolumn{2}{|l|}{ Lymph node metastasis } \\
\hline Yes & $72(71 \%)$ \\
\hline No & $30(29 \%)$ \\
\hline \multicolumn{2}{|c|}{ Loco-regional lymph node metastasis } \\
\hline Yes & $31(30 \%)$ \\
\hline No & $71(70 \%)$ \\
\hline \multicolumn{2}{|l|}{ Liver metastasis } \\
\hline Yes & $19(19 \%)$ \\
\hline No & $83(81 \%)$ \\
\hline \multicolumn{2}{|l|}{ Brain metastasis } \\
\hline Yes & $14(14 \%)$ \\
\hline No & $88(86 \%)$ \\
\hline \multicolumn{2}{|l|}{ Malignant pleural effusion } \\
\hline Yes & $9(9 \%)$ \\
\hline No & $93(91 \%)$ \\
\hline \multicolumn{2}{|l|}{ Bone metastasis } \\
\hline Yes & $27(26 \%)$ \\
\hline No & 75 (74\%) \\
\hline \multicolumn{2}{|l|}{ Adrenal gland metastasis } \\
\hline Yes & $11(11 \%)$ \\
\hline No & $91(89 \%)$ \\
\hline \multicolumn{2}{|l|}{ N. of metastatic sites } \\
\hline 1 & $21(21 \%)$ \\
\hline 2 & 34 (33\%) \\
\hline 3 & 47 (46\%) \\
\hline
\end{tabular}

metastases corresponds to 20 points, the presence of liver metastases corresponds to 0 points, while the ECOG PS of 1 corresponds to 50 points. The total point of 70 corresponds a 3- and 6-month OS of about $0.6(60 \%)$ and 0.3 (30\%), respectively.

The C-indexes for OS models was 0.76 and calibration of the nomogram for OS was considered adequate (Fig. 4). Kaplan-Meier curves according to the range of

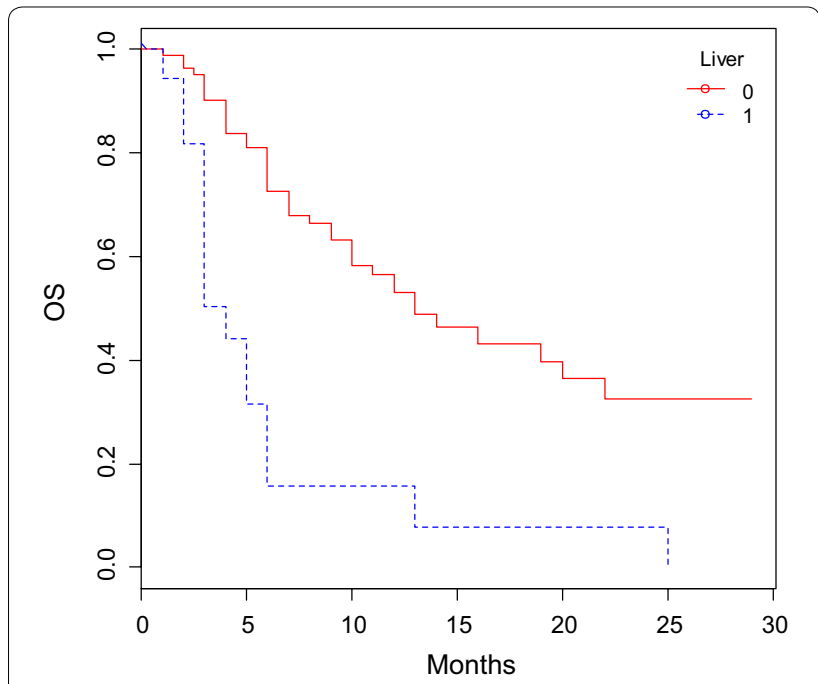

Fig. 1 OS according to liver metastases status

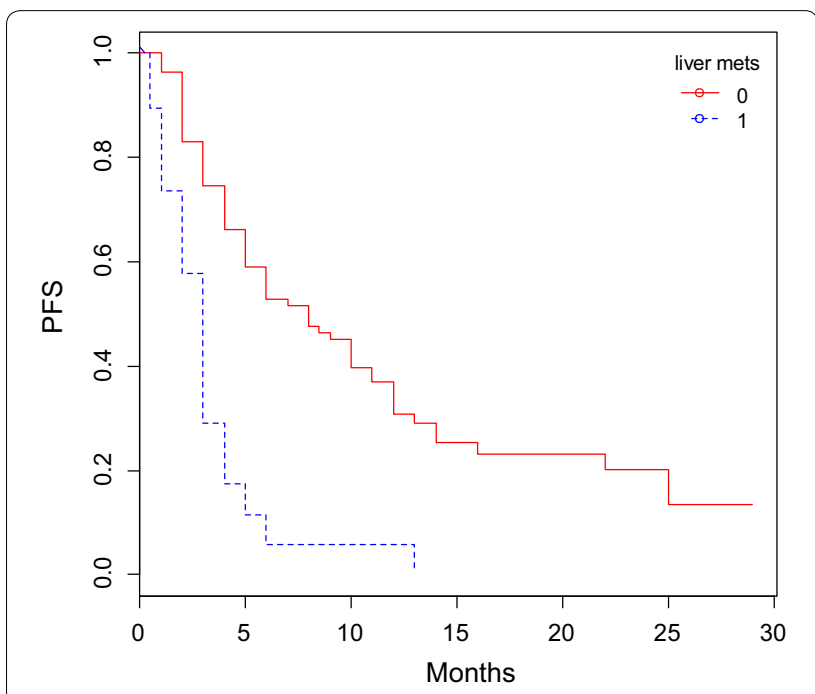

Fig. 2 PFS according to liver metastases status

total points highlighted the appropriateness of distinguish the patients' survival in all the subgroups (Fig. 5). The groups were obtained considering the total point distribution of our population. Group I and II (red lines) represent patients with poor outcome.

The PFS was significantly shorter in patients with liver metastases $(P<0.0001)$ and higher ECOG PS score $(P<0.0001)$, while was longer in patients having only lung lymph nodes $(P=0.0017)$ and lung metastases $(P=0.0443)$. ECOG PS $(P<0.001)$ and liver metastases $(P<0.001)$ resulted statistically significant at MVA. The $\mathrm{C}$-indexes for PFS models was 0.72 . 
Table 2 Univariate and multivariate analysis for OS

\begin{tabular}{|c|c|c|c|c|}
\hline \multirow[t]{2}{*}{ Covariate } & \multicolumn{2}{|l|}{ Univariate analysis } & \multicolumn{2}{|l|}{ Multivariate analysis } \\
\hline & $\mathrm{HR}(95 \% \mathrm{Cl})$ & P value & $\mathrm{HR}(95 \% \mathrm{Cl})$ & P value \\
\hline Age $\geq 69$ years & $0.6389(0.3691-1.1060)$ & 0.057 & - & - \\
\hline Sex & $1.4(0.7892-2.485)$ & 0.247 & - & - \\
\hline Weight $\geq 69.5 \mathrm{~kg}$ & $1.1999(0.6606-2.1794)$ & 0.543 & - & - \\
\hline Height $>168 \mathrm{~cm}$ & $0.9492(0.4884-1.8445)$ & 0.876 & - & - \\
\hline Liver metastasis & $3.6912(2.0123-6.7710)$ & 0.000 & $3.0637(1.6693-.623)$ & 0.0003 \\
\hline Lung metastasis & $0.4954(0.2858-0.8587)$ & 0.017 & $0.6209(0.3564-1.082$ & 0.0925 \\
\hline Lymph node metastasis & $0.4954(0.2858-0.8587)$ & 0.2723 & - & - \\
\hline Only lymph node metastasis & $0.4482(0.2363-0.8502)$ & 0.0145 & - & - \\
\hline Brain metastasis & $0.9460(0.4728-1.8927)$ & 0.876 & - & - \\
\hline Malignant pleural effusion & & 0.991 & - & - \\
\hline Bone metastasis & $1.4029(0.8022-2.4535)$ & 0.2375 & - & - \\
\hline Adrenal gland metastasis & $1.1271(0.5095-2.4933)$ & 0.7689 & - & - \\
\hline N. of metastatic sites & $1.1610(0.7966-1.6921)$ & 0.4395 & - & - \\
\hline ECOG PS & $2.706(1.774-4.126)$ & $<0.0001$ & $2.588(1.655-4.046)$ & $<0.0001$ \\
\hline Line of treatment & $1.1507(0.7571-1.7488)$ & 0.5133 & - & - \\
\hline Global & & & & $<0.0001$ \\
\hline
\end{tabular}

Italic values refer to statistically significant covariates $(p$ value $<0.05)$ for OS

Table 3 Univariate and multivariate analysis for PFS

\begin{tabular}{|c|c|c|c|c|}
\hline \multirow[t]{2}{*}{ Covariate } & \multicolumn{2}{|l|}{ Univariate analysis } & \multicolumn{2}{|c|}{ Multivariate analysis } \\
\hline & HR $(95 \% \mathrm{Cl})$ & $P$ value & $\mathrm{HR}(95 \% \mathrm{Cl})$ & $P$ value \\
\hline Age ( $\geq 69$ years) & $0.7437(0.4732-1.1688)$ & 0.2015 & - & - \\
\hline Sex & $1.313(0.8109-2.126)$ & 0.268 & - & - \\
\hline Weight ( $\geq 69.5$ kg) & 1.1089 (0.6850-1.7950) & 0.6756 & - & - \\
\hline Height (> 168 cm) & $1.0502(0.6274-1.7577)$ & 0.8530 & - & - \\
\hline Liver metastasis & $2.9391(1.6952-5.0957)$ & 0.0001 & $3.456(2.002-5.965)$ & $<0.0001$ \\
\hline Lung metastasis & $0.6133(0.3818-0.9852)$ & 0.0443 & - & - \\
\hline Lymph node metastasis & $0.7610(0.4697-1.2330)$ & 0.2697 & - & - \\
\hline Only Lymph node metastasis & $0.4324(0.2567-0.7282)$ & 0.0017 & - & - \\
\hline Brain metastasis & $1.0419(0.5639-1.9251)$ & 0.8964 & - & - \\
\hline Malignant pleural effusion & $1.0225(0.4452-2.3485)$ & 0.9583 & - & - \\
\hline Bone metastasis & $1.5459(0.9538-2.5057)$ & 0.0786 & - & - \\
\hline Adrenal gland metastasis & $1.2286(0.6145-2.4563)$ & 0.5623 & - & - \\
\hline N. of metastatic sites & $1.1524(0.8550-1.5533)$ & 0.3542 & - & - \\
\hline ECOG PS & $2.26(1.602-3.187)$ & $<0.0001$ & $2.260(1.580-3.233)$ & $<0.0001$ \\
\hline Line of treatment & $1.0253(0.7140-1.4724)$ & 0.8928 & - & - \\
\hline Global & & & & $<0.0001$ \\
\hline
\end{tabular}

Italic values refer to statistically significant covariates $(p$ value $<0.05)$ for PFS

\section{Discussion}

In the last few years, an unprecedented number of immunotherapeutics including the anti-PD1 nivolumab have proven effective compared to standard chemotherapy in pretreated NSCLC patients that has greatly expanded treatment options beyond first-line. However, in spite of a proportion of them deriving a long-term disease control, roughly $60-80 \%$ of patients progress on ICI carrying a dismal prognosis [18]. Hence, the question on how to properly select the best candidates to second-line immunotherapy has rapidly risen and represents thus an urgent unmet need. 


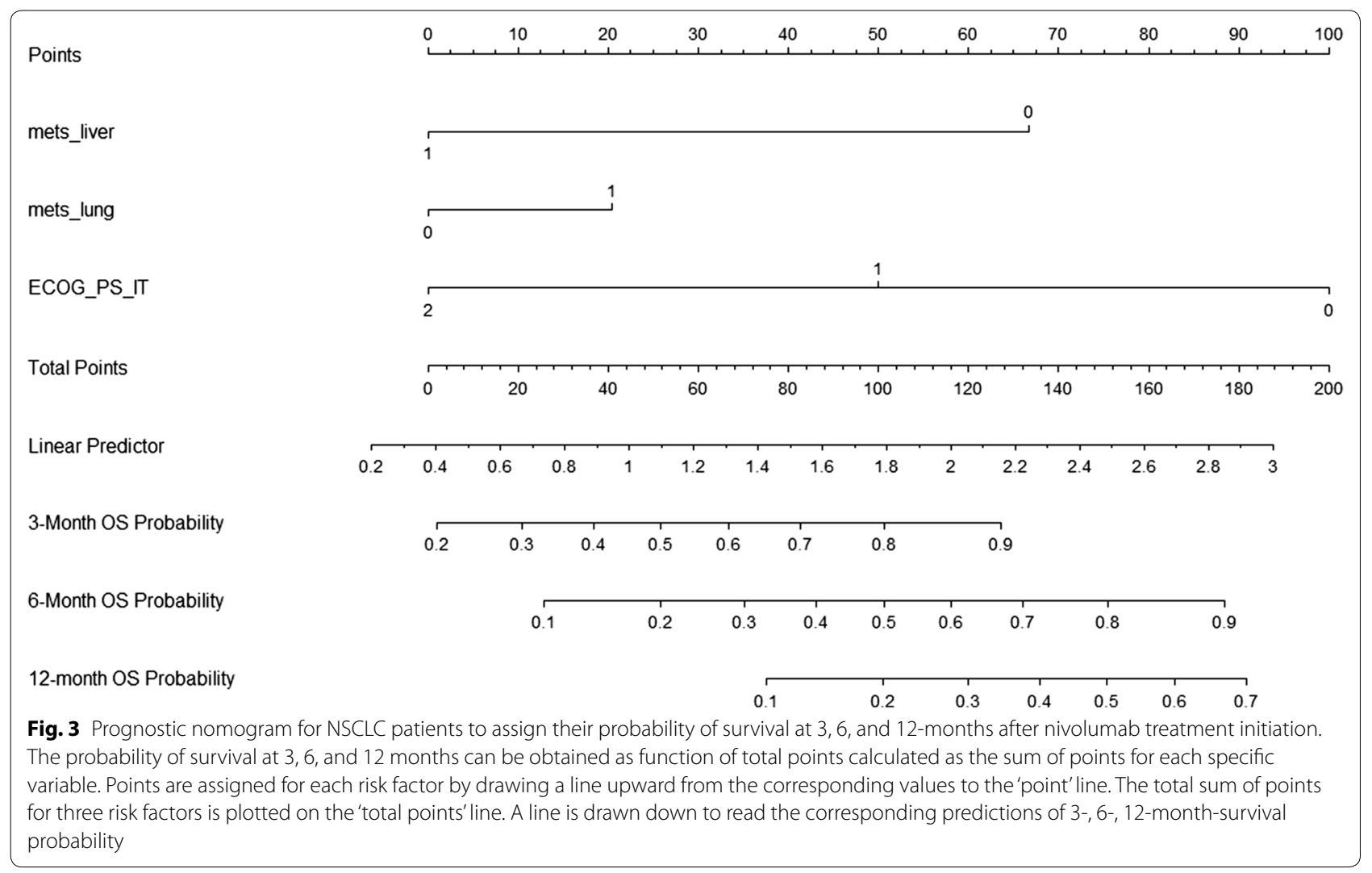

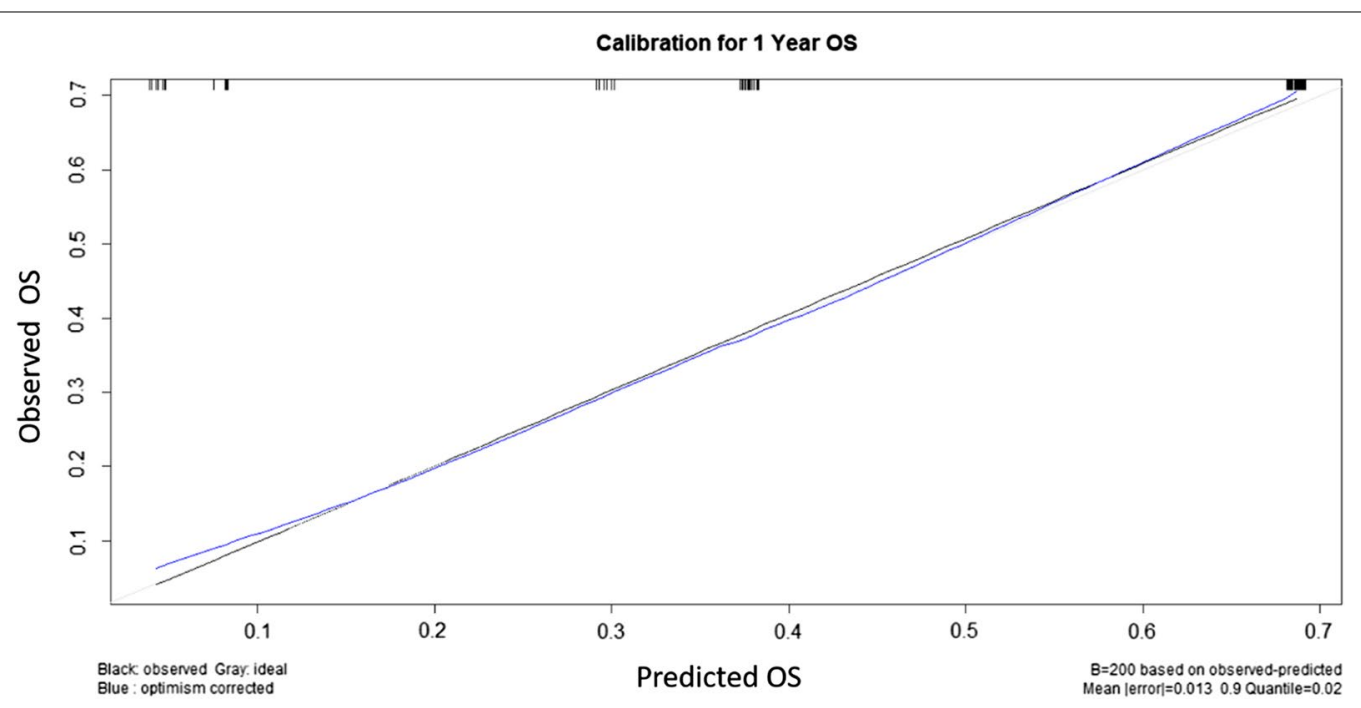

Fig. 4 Calibration plot of the final nomogram for OS. All patients were grouped based on their predicted probabilities. Mean predicted probabilities were plotted against the actual incidence of PFS. The reference line represents perfect quality of observed frequencies and predicted probabilities

Of interest, some reports have proposed clinical prognostic factors in advanced-stage NSCLC treated with second-line nivolumab, among which is ECOG PS.
Our findings concerning the impact of patients' general health conditions on nivolumab efficacy are aligned with those previously described in the literature. Indeed, poor ECOG PS $(\geq 2)$ has been consistently shown to be 


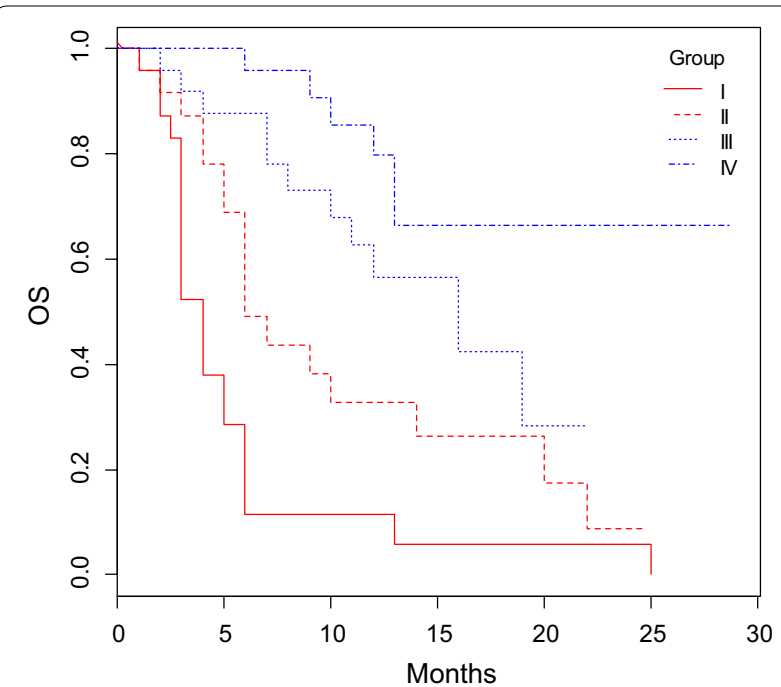

group I: $78.4-151.4$

group II: $151.4-173.5$

group III: $173.5-197.65$

group IV: $197.65-223$

Fig. 5 OS according to the nomogram total points (I-IV ranges). The groups were obtained considering the total point distribution of our population. Group I and II (red lines) represent patients with poor outcome

an independent predictor of poorer survival in both clinical trials and real-life experience of nivolumab-treated patients [19-21]. With regards to the role of age, at the UVA, we found that elderly patients ( $>70$ years) were less likely than younger to benefit from nivolumab. Limited and conflicting data are available regarding the safety and efficacy of nivolumab in older people due to their underrepresentation in clinical trials and the lack of randomized studies in this specific subset of patients.

Among disease characteristics in our cohort, lung and lymph nodes metastases seemed to be favourable prognostic factors, while liver involvement emerged as a negative feature. Accordingly, a growing number of studies have been suggesting that ICI treatment may have a differential outcome based on the type of metastatic involvement. In particular, liver metastases have been independently associated with poorer survival in the real-world setting $[16,17]$. Likewise, the updated follow-up of CheckMate 017 and CheckMate 057 demonstrated in the experimental arm 3-year OS of $8 \%$ vs. $17 \%$ in patients with liver metastases compared to the whole population [18]. Consistently, a cohort from Keynote 001 evaluating another anti-PD1 pembrolizumab confirmed a reduced response rate $(28.6 \%$ vs. $56.7 \%)$ and shortened PFS in NSCLC patients with liver metastasis (mPFS 1.8 vs. 4 months, $\mathrm{P}=0.0094$ ), compared to those without liver metastasis [22]. To this end, accumulating preclinical evidence is shedding light on the differential response and clinical benefit seen with anti-PD1 agents according to metastatic sites and specifically on the poorer prognosis of patients with liver metastases.

It was demonstrated that liver metastases seem to be "colder" than a primary tumour or lung and lymph node metastases [23]. In addition, in the liver microenvironment, $\mathrm{T}$ cells have been shown to interact with sinusoidal endothelial cells, resulting in differentiation of the $\mathrm{T}$ cells into a Treg phenotype and/or in partial activation of the $\mathrm{T}$ cells, followed by passive cell death [24]. More interestingly, circulating levels of Eotaxin-2 and IP-10 that are chemokines attracting immunosuppressive immune cells have been reported to be higher in patients with liver metastatic involvement from both melanoma and colorectal cancer, further pointing toward unique immunosuppressive mechanisms sustained by liver metastases.

In the present study, we investigated the role of clinical features in order to build a nomogram enabling individualized OS estimation in a real-world cohort of advanced NSCLC receiving the anti-PD1 agent nivolumab as second- or later line of treatment. The prognostic nomogram is based on readily available, inexpensive and easily-to-collect patient (ECOG PS) and disease variables (liver and lung metastases).

The present study has some limitations to be acknowledged. This is a retrospective bicentric cohort study with a 11-month follow up and a relatively small sample size, thereby with potential for inherent biases. Still, the lack of data regarding PD-L1 expression determined by using the Tumor Proportion Score is another shortcoming of the analysis. Finally, external prospective validation is required to assess reproducibility and generalizability of our results.

\section{Conclusion}

We developed an easy-to-use and inexpensive device to assist the clinician with a quantitative tool to predict OS probability in NSCLC treated with nivolumab in the clinical practice. The nomogram was built on the basis of clinico-pathological variables which retained independent prognostic value in the MVA and showed an adequate performance. While waiting for novel biomarkers, this prognostic tool could be valuable to more accurately driving treatment decision in daily practice and enrollment onto clinical trials.

\section{Authors' contributions}

$A B, M S$ : conceptualization, data curation, formal analysis, investigation, writing - original draft preparation. LS, data analysis, writing and interpretation. FRDP, BC, IGZ, RG, MM, FM, MR, PV, LP, MN: data curation, investigation. PM: project administration, supervision, writing —original draft preparation, writing-review and editing. All authors read and approved the final manuscript. 


\section{Author details}

${ }^{1}$ Department of Clinical and Molecular Medicine, Sant'Andrea Hospital, Sapienza University of Rome, Rome, Italy. ${ }^{2}$ Department of Oncology, University Hospital of Modena and Reggio Emilia, Modena, Italy. ${ }^{3}$ Division of Molecular Pathology, The Institute of Cancer Research and Gastrointestinal Unit, The Royal Marsden Hospital, London, Sutton, UK. ${ }^{4}$ Laboratory of Medical Physics and Expert Systems, Regina Elena National Cancer Institute, Rome, Italy. ${ }^{5}$ Department of Radiological, Oncological and Pathological Sciences, Sapienza University of Rome, Rome, Italy. ${ }^{6}$ Department of Experimental Medicine, Sapienza University of Rome, Rome, Italy. ${ }^{7}$ Division of Medical Oncology 2, IRCCS Regina Elena National Cancer Institute, Rome, Italy.

\section{Acknowledgements}

None.

\section{Competing interests}

The authors declare that they have no competing interests.

\section{Availability of data and materials}

All data generated or analysed during this study are included in this published article.

\section{Consent for publication}

Not applicable.

\section{Ethics approval and consent to participate}

The final version of the protocol was approved by the Institutional Ethics Committee of the two Institutions involved. All patients gave written informed consent.

\section{Funding}

None.

\section{Publisher's Note}

Springer Nature remains neutral with regard to jurisdictional claims in published maps and institutional affiliations.

Received: 2 January 2019 Accepted: 12 March 2019

Published online: 27 March 2019

\section{References}

1. Bray F, Ferlay J, Soerjomataram I, et al. Global cancer statistics 2018 : GLOBOCAN estimates of incidence and mortality worldwide for 36 cancers in 185 countries. CA Cancer J Clin. 2018;68(6):394-424. https:// doi.org/10.3322/caac.21492 Epub 2018 Sep 12

2. Planchard D, Popat S, Kerr K, et al. Metastatic non-small cell lung cancer: ESMO Clinical Practice Guidelines for diagnosis, treatment and follow-up. Ann Oncol. 2018;29(Supplement_4):iv192-237. https://doi.org/10.1093/ annonc/mdy275.

3. Raju S, Joseph R, Sehgal S. Review of checkpoint immunotherapy for the management of non-small cell lung cancer. Immunotargets Ther. 2018;31(7):63-75. https://doi.org/10.2147/ITT.S125070 (eCollection 2018. Review)

4. Salati M, Baldessari C, Cerbelli B, et al. Nivolumab in pretreated non-small cell lung cancer: continuing the immunolution. Transl Lung Cancer Res. 2018;7(Suppl 2):S91-4. https://doi.org/10.21037/tlcr.2018.01.14.

5. Reck $M$, Rodriguez-Abreu $D$, Robinson $A G$, et al. Pembrolizumab versus chemotherapy for PD-L1-positive non-small-cell lung cancer. N Engl J Med. 2016:375:1823-33.

6. Gandhi L, Rodriguez-Abreu D, Gadgeel S, et al. Pembrolizumab plus chemotherapy in metastatic non-small-cell lung cancer. N Engl J Med. 2018:378:2078-92.
7. Socinski MA, Jotte RM, Cappuzzo F, et al. Atezolizumab for firstline treatment of metastatic nonsquamous NSCLC. N Engl J Med. 2018:378:2288-301.

8. Papadimitrakopoulou V, Cobo M, Bordon R et al. IMPOWER132: PFS and safety results with $1 \mathrm{~L}$ atezolizumab + carboplatin/cisplatin + pemetrexed in stage IV non-squamous NSCLC. In: IASLC 19th world conference on lung cancer 2018; abstr. OA05.07.

9. Brahmer J, Reckamp KL, Baas P, et al. Nivolumab versus docetaxel in advanced squamous-cell non-small-cell lung cancer. N Engl J Med. 2015;373:123-35

10. Borghaei H, Paz-Ares L, Horn L, et al. Nivolumab versus docetaxel in advanced nonsquamous non-small-cell lung cancer. N Engl J Med. 2015;373:1627-39.

11. Herbst RS, Baas P, Kim DW, et al. Pembrolizumab versus docetaxel for previously treated, PD-L1-positive, advanced non-small-cell lung cancer (KEYNOTE-010): a randomised controlled trial. Lancet. 2016:387:1540-50.

12. Shukuya T, Carbone DP. Predictive markers for the efficacy of anti-PD-1/ PD-L1 antibodies in lung cancer. J Thorac Oncol. 2016;11(7):976-88. https ://doi.org/10.1016/j.jtho.2016.02.015 (Epub 2016 Mar 2. Review).

13. Lo Russo G, Moro M, Sommariva M, et al. Antibody-Fc/FcR interaction on macrophages as a mechanism for hyperprogressive disease in non-small cell lung cancer subsequent to PD-1/PD-L1 blockade. Clin Cancer Res. 2018. https://doi.org/10.1158/1078-0432.ccr-18-139.

14. Voong KR, Feliciano J, Becker D. Levy B beyond PD-L1 testing-emerging biomarkers for immunotherapy in non-small cell lung cancer. Ann Transl Med. 2017;5(18):376. https://doi.org/10.21037/atm.2017.06.48.

15. Botticelli A, Cerbelli B, Lionetto $L$, et al. Can IDO activity predict primary resistance to anti-PD-1 treatment in NSCLC? J Transl Med. 2018;16(1):219. https://doi.org/10.1186/s12967-018-1595-3.

16. Tamiya M, Tamiya A, Inoue T, et al. Metastatic site as a predictor of nivolumab efficacy in patients with advanced non-small cell lung cancer: a retrospective multicenter trial. PLoS ONE. 2018;13(2):e0192227. https:// doi.org/10.1371/journal.pone.0192227 (eCollection 2018).

17. Garde-Noguera J, Martin-Martorell P, De Julián M, et al. Predictive and prognostic clinical and pathological factors of nivolumab efficacy in non-small-cell lung cancer patients. Clin Transl Oncol. 2018;20(8):1072-9. https://doi.org/10.1007/s12094-017-1829-5 (Epub 2018 Jan 24)

18. Vokes EE, Ready N, Felip E, et al. Nivolumab versus docetaxel in previously treated advanced non-small-cell lung cancer (CheckMate 017 and CheckMate 057): 3-year update and outcomes in patients with liver metastases. Ann Oncol. 2018;29(4):959-65. https://doi.org/10.1093/annonc/mdy041.

19. Peters $S$, Creelan $B$, Hellmann MD et al. CT082 -impact of tumor mutation burden on the efficacy of first-line nivolumab in stage iv or recurrent non-small cell lung cancer: Aan exploratory analysis of CheckMate 026, AACR Annual Meeting, Washington, DC. 2017.

20. Dudnik E, Moskovitz M, Daher S, et al. Effectiveness and safety of nivolumab in advanced non-small cell lung cancer: the real-life data. Lung Cancer. 2017;56:78. https://doi.org/10.1016/j.lungcan.2017.11.015 ((Epub ahead of print))

21. Spigel D, Schwartzberg L, Waterhouse D, Hussein M, Jotte R, Stepanski E, et al. Is nivolumab safe and effective in elderly and PS2 patients with nonsmall cell lung cancer (NSCLC)? Results of CheckMate 153. In: Presented at the international association for the study of lung cancer 17th world conference on lung cancer, Vienna, Austria. 2016. Poster P3.02ce026.

22. Tumeh PC, Hellmann MD, Hamid $O$, et al. Liver metastasis and treatment outcome with anti-pd-1 monoclonal antibody in patients with melanoma and NSCLC. Cancer Immunol Res. 2017. https://doi. org/10.1158/2326-6066.cir-16-0325.

23. Li Y, Chang C-W, Tran D, et al. Prevalence of PDL1 and tumor infiltrating lymphocytes (TILs) in primary and metastatic TNBC. Cancer Res. 2018;78(4 Supplement):PD6-01.

24. Crispe IN. Hepatic T cells and liver tolerance. Nat Rev Immunol. 2003;3(1):51-62. 